Resumen por el autor, Bennet M. Allen.

Universidad de Kansas.

Desarrollo de las glándulas tiroides de Bufo y su relación normal con la metamorfosis.

La acumulación de material coloide en las glándulas tiroides de los renacuajos de sapo coincide con la aparición de los rudimentos de los miembros posteriores. Las masas coloides aumentan en tamaño y número hasta que los miembros anteriores perforan la piel. Esta acumulación de material coloide está acompañada de un marcado aumento de tamaño en las glándulas, el cual parece ser un resultado directo de aquella. El hecho aparentemente paradójico de la cesación de crecimiento y actual disminución de tamaño de las glándulas tiroides y de las masas coloides en el momento en que el proceso de la metamorfosis es mas activo, podría explicarse en parte como el resultadó de un proceso parcial de desecación debido a la emergencia de los renacuajos fuera del agua, si la salida de estos fuera de dicho medio no tuviese lugar en un estado ulterior. La reducción de tamaño está pues, realizándose antes de que el factor citado pueda ser efectivo. Es mucho mas probable que tal disminución se deba a la absorción por la sangre de una cantidad considerable del coloide almacenado en las gládulas, en el momento en que dicho material puede producir mas efectos. La cola aumenta continuamente de tamaño hasta un cierto momento, presenta una ligera, disminución y desaparece después rápidamente. La secrección tiroidla no es causa de la disminución de tamaño de la cola ni alcanza unvolúmen considerable antes de ser suficiente para producir tal resultado. Es indudable que el desarrollo de los miembros y el proceso de desaparición de la cola siguen a la acumulación de coloide en las glándulas tiroideas de Bufo.

Translation by José F. Nonidez

Carnegie Institution of Washington 
A UTHOR'S ABSTRACT OF THIS PAPER ISSUED

BY THE BIBLIOGRAPHIO SERVIGE, AUGUST 11

\section{THE DEVELOPMENT OF THE THYREOID GLANDS OF BUFO AND THEIR NORMAL RELATION TO METAMORPHOSIS ${ }^{\mathrm{I}}$ \\ BENNET M. ALLEN \\ Department of Zoology, University of Kansas}

ONE PLATE (SIX FIGURES) AND ONE TEXT FIGURE

In recent years much light has been thrown upon the influence of the thyreoid gland upon growth. Most striking are recent studies upon the influence that this gland exerts upon metamorphosis in the amphibians. Gudernatsch ('12) showed by experimental means that feeding thyreoid preparations of sheep to tadpoles of Rana greatly accelerated their metamorphosis. This experiment has been repeated by others (Swingle, '18) and completely verified. In 1916 the writer was successful in removing the thyreoid-gland anlagen from young tadpoles, which were then reared to a period long after that at which metamorphosis should normally occur, one tadpole being kept alive until fourteen months after the operation. In all successful cases there was total failure to metamorphose, although the hind limbs underwent a limited amount of growth and the fore limbs began to develop without, however, breaking through the skin.

IIoskins and Morris ('16) successfully accomplished the removal of the thyreoid gland in a similar way at the same time in Rana and Amblystoma. They had some difficulty in rearing the operated tadpoles, but were successful the following season. Their work was reported at the December, 1916, meeting of the American Association of Anatomists and again at the December, 1917, meeting.

In the spring of 1917 the writer repeated his earlier experiments upon Rana and also performed similar operations upon

${ }^{1}$ Contribution No. 318 from the Department of Zoology, University of Kansas. 
Bufo. These experiments clearly demonstrate that the thyreoid glands exert a marked influence upon development-an excess of thyreoid (feeding) accelerating metamorphosis and the removal of the thyreoid gland producing decided retardation of metamorphosis. It is thus seen that these two methods of investigation give results that are fully corroborative of one another.

There are certain points in the solution of this problem that can be best attacked by a study of the normal relation of these glands to metamorphosis. It was shown in Rana pipiens that the thyreoidless and normal control tadpoles show no differences up to the time when the limb buds begin to develop, but as soon as these have made their appearance in the thyreoidless tadpoles they show a marked retardation in growth, while the body continues to grow in size. As far as our observations go, it was found that all somatic features of tadpole development were thus retarded, the gonads alone being unaffected (Allen, Rogers and Terry, '18). In order properly to interpret investigations of this kind, it is necessary to have definite data regarding the normal relation of the thyreoid glands to development.

The development of the thyreoid glands of Anurans was described by Goette in Bombinator. A paper by W. Müller gave the first complete account of the process. This was worked out in Rana temporaria. Müller found that the thyreoid developed from a ventral downgrowth of the floor of the pharynx. This anlage was at first unpaired, but later became divided into two parts by the development of the copula of the hyoid cartilage. He traced the further development of the gland from a condition of a solid mass to a looser texture, accompanied by the disappearance of the pigment cells characteristic of the early stages, and he found later that it consists of a network of twisted cords which are surrounded by looser connective tissue. He traced the development through later stages in which vesicles had developed. These were composed of a single layer of epithelium and filled with colloid. He found that in young frogs immediately after metamorphosis, the thyreoid gland is wholly made up of the vesicles containing colloid. 
De Meuron ('86) also described the early stages in the development of the Anuran thyreoid glands.

Maurer ('88) worked out the development of the thyreoid gland in Rana esculenta. He found the division of the median anlage to take place in the 13-mm. larva. At this time the cells were deeply pigmented, loosely arranged, and showed the first beginnings of vesicle formation. His account of the further development did not take it up in great detail, but showed that by the time of metamorphosis the thyreoid was well developed, being composed of a large number of follicles containing colloid. This was very clearly illustrated in one of his beautiful figures. We thus see that the general development of the thyreoid has been pretty thoroughly worked out. It remains, however, to show the relation between thyreoid development and the general body features which become modified during metamorphosis. A study of this kind involves close attention to the length of body, length of tail, length of limb, etc., and a comparison of these features with the volume of the thyreoid at various stages of development. In order to have any force in showing the relation of the thyreoid gland to metamorphosis, this work must be done in a roughly quantitative fashion. None of the investigators up to the present time have attacked the problem from this angle with one exception. Leo Adler ('14) made a few observations upon the size of the thyreoid gland in different stages of Rana temporaria. His series was made up of one each, the length of the thyreoid gland being given in parenthesis after the total length dimensions of each stage. His measurements were as follows: $20 \mathrm{~mm}$. (0.07 mm.); $23 \mathrm{~mm}$. (0.09 mm.); $25 \mathrm{~mm}$. (0.10 mm.); $28 \mathrm{~mm}$. (0.16 mm.); $30 \mathrm{~mm}$. (0.21 mm.); $33 \mathrm{~mm}$. (0.32 mm.); $35 \mathrm{~mm}$. (0.28 mm.) ; $40 \mathrm{~mm}$. (0.24 mm.). He states that the $28-\mathrm{mm}$. tadpoles have hind legs which show a division into joints, but no statement is made as to their length. The 33-mm. tadpoles have completely developed hind legs, while the fore legs are visible through the skin. Adler states that the last two tadpoles (35 mm. and $40 \mathrm{~mm}$.) are abnormal in size. They had been hindered in metamorphosis, at first by temperature that was too high and, later, by temperature that was too low. 


\section{MATERIAL AND METHODS}

A very complete collection of tadpoles of Bufo lentiginosus gathered in Lawrence, Kansas, afforded all of the stages, from the first appearance of the hind-limb buds to the completion of metamorphosis. A number of specimens were fixed in Flemming's fluid and others in bichromate acetic. Large quantities of Bufo material fixed in 5 per cent formalin were used for a study of the gross features of the development of the thyreoid gland. These proved to be most valuable.

I wish to express my grateful acknowledgment of facilities and assistance accorded me by the Department of Anatomy of the University of Illinois Medical School. The greater part of the sections and illustrations used in this work were made by their technicians and artists. A series of gross dissections of Bufo were made in our University of Kansas Zoological Laboratory.

The sections were cut at a thickness of $10 \mu$ and were for the most part stained with haematoxylin and eosin. In some cases Heidenhain's iron alum-haematoxylin was used.

The dissections were made under a binocular microscope in such a fashion that the exposed thyreoid glands were left adherent to the hyoid cartilage, the whole being stained with alumcochineal. These dissections were dehydrated and then cleared in oil of wintergreen. They were preserved and finally studied in this fluid.

Measurements were made by means of a micrometer eyepiece, the maximum length, breadth, and thickness being determined in each ease. In making a measurement of the thickness, the hyoid cartilage was held vertical between two small pieces of glass and the extreme thickness was measured in optical section. The accompanying table 1 gives the measurements obtained from a study of the dissections just mentioned. Length, breadth, and thickness of the gland were multiplied together to give a rough approximation of the volume, in effect the volume of a parallelopiped that would contain the gland. The latter is flattened oval, somewhat irregular in some instances, but for the most part of relatively constant shape. 
TABLE 1

Table of measurements of Bufo lentiginosus larvae and newiy metamorphosed individuals

\begin{tabular}{|c|c|c|c|c|c|c|c|c|c|}
\hline \multirow{2}{*}{ NUMBER } & \multirow{2}{*}{$\begin{array}{l}\text { TOTAL } \\
\text { LENGTH }\end{array}$} & \multirow{2}{*}{$\begin{array}{c}\text { BODY } \\
\text { LENGTH }\end{array}$} & \multirow{2}{*}{$\begin{array}{c}\text { TAIL } \\
\text { LENGTH }\end{array}$} & \multirow{2}{*}{$\underset{\text { HIND LEG }}{\text { GENGTH }}$} & \multicolumn{3}{|c|}{ THYFEOID } & \multirow{2}{*}{ VOLUME } & \multirow{2}{*}{$\begin{array}{l}\text { AVERAGE } \\
\text { VOLUME }\end{array}$} \\
\hline & & & & & Length & Breadth & $\begin{array}{c}\text { Thick- } \\
\text { ness }\end{array}$ & & \\
\hline \multirow{3}{*}{ I a } & $m m$. & $m m$. & $m m$. & $m m$ & $m m$. & $m m$ & $m m$. & $\mathrm{cmm}$. & $\mathrm{cm} m$. \\
\hline & 21.6 & 9.7 & 11.9 & 1.386 & R. 0.273 & 0.119 & 0.091 & 0.0029 & \\
\hline & & & & & L. 0.322 & 0.112 & 0.091 & 0.0032 & 0.0030 \\
\hline \multirow[t]{2}{*}{ I b } & 21.1 & 9.6 & 11.5 & 1.320 & R. 0.217 & 0.112 & 0.077 & 0.0017 & \\
\hline & & & & & L. 0.189 & 0.112 & 0.084 & 0.0017 & 0.0017 \\
\hline \multirow[t]{2}{*}{$I c$} & 20.9 & 9.9 & 11.0 & 1.221 & R. 0.280 & 0.112 & 0.049 & 0.0015 & \\
\hline & & & & & L. 0.322 & 0.091 & 0.049 & 0.0014 & 0.0014 \\
\hline \multirow[t]{2}{*}{$I d$} & 20.1 & 9.6 & 10.5 & 1.353 & R. 0.385 & 0.147 & 0.084 & 0.0045 & \\
\hline & & & & & L. 0.315 & 0.119 & 0.077 & 0.0030 & 0.0037 \\
\hline \multirow[t]{2}{*}{$\mathrm{I} \mathrm{e}$} & 22.2 & 9.9 & 12.3 & 1.386 & R. 0.294 & 0.119 & 0.049 & 0.0017 & \\
\hline & & & & & L. 0.294 & 0.126 & 0.063 & 0.0021 & 0,0019 \\
\hline \multirow[t]{2}{*}{ If } & 21.1 & 9.9 & 11.2 & 1.188 . & $R .0 .301$ & 0.133 & 0.049 & 0.0019 & \\
\hline & & & & & L. 0.266 & 0.112 & 0.063 & 0.0018 & 0.0018 \\
\hline \multirow[t]{2}{*}{$I \mathrm{~g}$} & 20.8 & 10.0 & 10.8 & 1.254 & R. 0.308 & 0.126 & 0.035 & 0.0012 & \\
\hline & & & & & L. 0.280 & 0.091 & 0.035 & 0.0008 & 0.0010 \\
\hline \multirow[t]{2}{*}{$I \mathrm{~h}$} & 20.1 & 9.7 & 10.4 & 1.056 & R. 0.161 & 0.105 & 0.1028 & 0.0017 & \\
\hline & & & & & L. 0.203 & 0.119 & 0.035 & 0.0008 & 0.0013 \\
\hline \multirow[t]{2}{*}{$I i$} & 19.5 & 9.1 & 10.4 & 0.924 & R. 0.182 & 0.077 & 0.035 & 0.0005 & \\
\hline & & & & & L. 0.210 & 0.084 & 0.028 & 0.0005 & 0.0005 \\
\hline \multirow[t]{2}{*}{$I j$} & 20.4 & 9.5 & 10.9 & 1.056 & R. 0.245 & 0.091 & 0.042 & 0.0009 & \\
\hline & & & & & L. 0.294 & 0.133 & 0.035 & 0.0015 & 0.0012 \\
\hline \multirow[t]{2}{*}{ II a } & 26.2 & 11.8 & 14.4 & 4.521 & R. 0.399 & 0.231 & 0.154 & 0.0138 & \\
\hline & & & & & L. 0.483 & 0.182 & 0.182 & 0.0155 & 0.0146 \\
\hline \multirow[t]{2}{*}{ II $\mathrm{b}$} & 26.1 & 11.9 & 14.2 & 2.739 & R. 0.378 & 0.203 & 0.133 & 0.0099 & \\
\hline & & & & & L. 0.420 & 0.224 & 0.126 & 0.012 & 0.0109 \\
\hline \multirow[t]{2}{*}{ II c } & 25.0 & 11.1 & 13.9 & 4.026 & R. 0.378 & 0.231 & 0.105 & 0.0087 & \\
\hline & & & - & & L. 0.336 & 0.231 & 0.119 & 0.0094 & 0.0090 \\
\hline \multirow[t]{2}{*}{ II d } & 26.0 & 11.9 & 14.1 & 2.574 & R. 0.385 & 0.217 & 0.140 & 0.0117 & \\
\hline & & & & & L. 0.364 & 0.231 & 0.119 & 0.0109 & 0.0113 \\
\hline
\end{tabular}


TABLE 1-Continued

\begin{tabular}{|c|c|c|c|c|c|c|c|c|c|}
\hline \multirow{2}{*}{ NUMBER } & \multirow{2}{*}{$\begin{array}{l}\text { TOTAL } \\
\text { LENGTH }\end{array}$} & \multirow{2}{*}{$\begin{array}{c}\text { BODY } \\
\text { LENGTH }\end{array}$} & \multirow{2}{*}{$\begin{array}{c}\text { TAIL } \\
\text { LENGTH }\end{array}$} & \multirow{2}{*}{$\begin{array}{l}\text { RIND LEG } \\
\text { LENGTH }\end{array}$} & \multicolumn{3}{|c|}{ THYREOID } & \multirow{2}{*}{ VOLUME } & \multirow{2}{*}{$\begin{array}{l}\text { AVERAGE } \\
\text { VOLUME }\end{array}$} \\
\hline & & & & & Length & Breadth & $\begin{array}{c}\text { Thick- } \\
\text { ness }\end{array}$ & & \\
\hline \multirow{3}{*}{ II $\mathrm{e}$} & $m m$ & $m m$ & $m m$ & $m m$. & $m m$ & $m m$ & $m m$ & $\mathrm{cmm}$. & $\mathrm{cmm}$. \\
\hline & 25.8 & 12.1 & 13.7 & 2.673 & R. 0.315 & 0.182 & 0.112 & 0.0061 & \\
\hline & & & & & L. 0.385 & 0.196 & 0.112 & 0.0084 & 0.0072 \\
\hline \multirow[t]{2}{*}{ II $\mathrm{f}$} & 23.9 & 11.5 & 12.4 & 3.432 & R. 0.378 & 0.217 & 0.133 & 0.0108 & \\
\hline & & & & & L. 0.392 & 0.196 & 0.112 & 0.0086 & 0.0097 \\
\hline \multirow[t]{2}{*}{ II $\mathrm{g}$} & 26.2 & 12.8 & 13.4 & 4.389 & R. 0.336 & 0.210 & 0.147 & 0.0107 & \\
\hline & & & & & L. 0.315 & 0.224 & 0.182 & 0.0123 & 0.0115 \\
\hline \multirow[t]{2}{*}{ II $h$} & 25.2 & 11.0 & 14.2 & 4.818 & R. 0.364 & 0.252 & 0.168 & 0.0153 & \\
\hline & & & & & L. 0.651 & 0.315 & 0.182 & 0.0363 & 0.0258 \\
\hline \multirow[t]{2}{*}{ II $\mathrm{i}$} & 25.1 & 11.2 & 13.9 & 2.640 & R. 0.420 & 0.231 & 0.133 & 0.0102 & \\
\hline & & & & & L. 0.378 & 0.161 & 0.122 & 0.0074 & 0.0088 \\
\hline \multirow[t]{2}{*}{ II j } & 25.8 & 11.2 & 14.6 & 4.884 & R. 0.385 & 0.203 & 0.161 & 0.0123 & \\
\hline & & & & & L. 0.497 & 0.266 & 0.175 & 0.0222 & 0.0172 \\
\hline \multirow[t]{2}{*}{ III a } & 27.2 & 12.2 & 14.0 & 7.227 & R. 0.504 & 0.308 & 0.206 & 0.0325 & \\
\hline & & & & & I. 0.434 & 0.266 & 0.168 & 0.0197 & 0.0261 \\
\hline \multirow[t]{2}{*}{ III $b$} & 25.7 & 12.4 & 13.3 & 7.326 & R. 0.525 & 0.315 & 0.217 & 0.0355 & \\
\hline & & & & & L. 0.511 & 0.301 & 0.161 & 0.0245 & 0.300 \\
\hline \multirow[t]{2}{*}{ III c } & 26.0 & 12.1 & 13.9 & 7.194 & R. 0.462 & 0.273 & 0.175 & 0.0211 & \\
\hline & & & & & L. 0.455 & 0.287 & 0.189 & 0.0248 & 0.230 \\
\hline \multirow[t]{2}{*}{ III d } & 27.2 & 12.2 & 15.0 & 7.194 & R. 0.455 & 0.301 & 0.189 & 0.0256 & \\
\hline & & & & & L. 0.406 & 0.301 & 0.168 & 0.0209 & 0.0232 \\
\hline \multirow[t]{2}{*}{ III e } & 27.1 & 12.4 & 14.7 & 8.283 & R. 0.413 & 0.301 & 0.182 & 0.0211 & \\
\hline & & & & & L. 0.455 & 0.308 & 0.189 & 0.0256 & 0.0233 \\
\hline \multirow[t]{2}{*}{ III $\mathrm{f}$} & 26.9 & 11.2 & 15.7 & 8.283 & R. 0.427 & 0.294 & 0.196 & 0.0249 & \\
\hline & & & & & L. 0.511 & 0.294 & 0.245 & 0.0355 & 0.0302 \\
\hline \multirow[t]{2}{*}{ III $\mathrm{g}$} & 27.1 & 12.4 & 14.7 & 7.161 & R. 0.476 & 0.322 & 0.182 & 0.0276 & \\
\hline & & & & & L. 0.441 & 0.329 & 0.189 & 0.0276 & 0.0276 \\
\hline \multirow[t]{2}{*}{ III $h$} & 28.1 & 12.2 & 15.9 & 7.524 & R. 0.420 & 0.294 & 0.182 & 0.0219 & \\
\hline & & & & & L. 0.399 & 0.252 & 0.175 & 0.0170 & 0.0194 \\
\hline \multirow[t]{2}{*}{ III i } & 27.8 & 11.9 & 15.9 & 8.679 & R. 0.518 & 0.350 & 0.224 & 0.0400 & \\
\hline & & & & & L. 0.238 & 0.154 & 0.119 & 0.0043 & 0.0221 \\
\hline
\end{tabular}


TABLE 1-Continued

\begin{tabular}{|c|c|c|c|c|c|c|c|c|c|}
\hline \multirow{2}{*}{ NUMBER } & \multirow{2}{*}{$\begin{array}{c}\text { TOTAL } \\
\text { LENGTH }\end{array}$} & \multirow{2}{*}{$\begin{array}{l}\text { BORY } \\
\text { IEAfrytr }\end{array}$} & \multirow{2}{*}{$\begin{array}{c}\text { TAIL } \\
\text { LENGTH }\end{array}$} & \multirow{2}{*}{$\begin{array}{c}\text { HIND LEG } \\
\text { LENGTH }\end{array}$} & \multicolumn{3}{|c|}{ THYREOID } & \multirow{2}{*}{ VOLCME } & \multirow{2}{*}{$\begin{array}{l}\text { AVERAOH } \\
\text { VOLUME }\end{array}$} \\
\hline & & & & & Length & Breadth & $\begin{array}{c}\text { Thick- } \\
\text { ness }\end{array}$ & & \\
\hline III j & $\begin{array}{l}m m \\
27.1\end{array}$ & $\begin{array}{l}m m . \\
11.7\end{array}$ & $\begin{array}{l}m m . \\
15.4\end{array}$ & $\begin{array}{c}m m . \\
8.745\end{array}$ & $\begin{array}{c}m m . \\
\text { R. } 0.427 \\
\text { L. } 0.409\end{array}$ & $\begin{array}{c}m m \\
0.329 \\
0.357\end{array}$ & $\begin{array}{c}m m . \\
0.259 \\
0.238\end{array}$ & $\begin{array}{c}c m m \\
0.0369 \\
0.0354\end{array}$ & $\begin{array}{c}c m m . \\
0.0361\end{array}$ \\
\hline IV a & 23.1 & 10.9 & 12.2 & 9.636 & $\begin{array}{r}\text { R. }\left\{\begin{array}{l}0.392 \\
0.280\end{array}\right. \\
\text { L. } 0.371\end{array}$ & $\begin{array}{l}0.308 \\
0.147 \\
0.350\end{array}$ & $\begin{array}{l}0.206 \\
0.210\end{array}$ & $\begin{array}{l}0.053 \\
0.272\end{array}$ & 0.0263 \\
\hline IV b & 25.3 & 10.5 & 14.8 & 9.702 & $\begin{array}{l}\text { R. } 0.385 \\
\text { L. } 0.392\end{array}$ & $\begin{array}{l}0.273 \\
0.266\end{array}$ & $\begin{array}{l}0.154 \\
0.133\end{array}$ & $\begin{array}{l}0.0154 \\
0.0137\end{array}$ & 0.0145 \\
\hline IV $\mathbf{c}$ & 23.6 & 10.6 & 13.0 & 8.943 & $\begin{array}{l}\text { R. } 0.413 \\
\text { L. } 0.427\end{array}$ & $\begin{array}{l}0.259 \\
0.259\end{array}$ & $\begin{array}{l}0.168 \\
0.168\end{array}$ & $\begin{array}{l}0.0181 \\
0.0190\end{array}$ & 0.0185 \\
\hline IV d & 25.4 & 11.3 & 14.1 & 9.306 & $\begin{array}{l}\text { R. } 0.574 \\
\text { L. } 0.623\end{array}$ & $\begin{array}{l}0.343 \\
0.322\end{array}$ & $\begin{array}{l}0.210 \\
0.206\end{array}$ & $\begin{array}{l}0.0407 \\
0.0418\end{array}$ & 0.0412 \\
\hline IV e & 26.8 & 11.9 & 14.9 & 9.966 & $\begin{array}{l}\text { R. } 0.567 \\
\text { L. } 0.609\end{array}$ & $\begin{array}{l}0.364 \\
0.385\end{array}$ & $\begin{array}{l}0.210 \\
0.206\end{array}$ & $\begin{array}{l}0.0433 \\
0.0487\end{array}$ & 0.0460 \\
\hline $\mathrm{IV} \mathbf{f}$ & 26.5 & 12.0 & 14.5 & 8.778 & $\begin{array}{l}\text { R. } 0.497 \\
\text { L. } 0.525\end{array}$ & $\begin{array}{l}0.322 \\
0.343\end{array}$ & $\begin{array}{l}0.210 \\
0.224\end{array}$ & $\begin{array}{l}0.0336 \\
0.0389\end{array}$ & 0.0362 \\
\hline IV $\mathrm{g}$ & 25.0 & 11.3 & 13.7 & 8.943 & $\begin{array}{l}\text { R. } 0.518 \\
\text { L. } 0.525\end{array}$ & $\begin{array}{l}0.329 \\
0.266\end{array}$ & $\begin{array}{l}0.175 \\
0.154\end{array}$ & $\begin{array}{l}0.0292 \\
0.0211\end{array}$ & 0.0251 \\
\hline IV $\mathrm{h}$ & 25.6 & 11.5 & 14.1 & 10.131 & $\begin{array}{l}\text { R. } 0.504 \\
\text { L. } 0.490\end{array}$ & $\begin{array}{l}0.357 \\
0.315\end{array}$ & $\begin{array}{l}0.217 \\
0.175\end{array}$ & $\begin{array}{l}0.0396 \\
0.0258\end{array}$ & 0.0327 \\
\hline IV i & 24.6 & 10.4 & 14.2 & 8.844 & $\begin{array}{l}\text { R. } 0.532 \\
\text { L. } 0.490\end{array}$ & $\begin{array}{l}0.322 \\
0.315\end{array}$ & $\begin{array}{l}0.208 \\
0.231\end{array}$ & $\begin{array}{l}0.0406 \\
0.0349\end{array}$ & 0.0377 \\
\hline IV $\cdot j$ & 21.7 & 10.9 & 10.8 & 7.448 & $\begin{array}{l}\text { R. } 0.511 \\
\text { L. } 0.560\end{array}$ & $\begin{array}{l}0.315 \\
0.336\end{array}$ & $\begin{array}{l}0.206 \\
0.217\end{array}$ & $\begin{array}{l}0.0332 \\
0.0419\end{array}$ & 0.0375 \\
\hline $\mathrm{V} \mathbf{a}$ & 12.9 & 11.6 & 1.3 & 10.956 & $\begin{array}{l}\text { R. } 0.385 \\
\text { L. } 0.413\end{array}$ & $\begin{array}{l}0.329 \\
0.301\end{array}$ & $\begin{array}{l}0.182 \\
0.210\end{array}$ & $\begin{array}{l}0.0226 \\
0.0258\end{array}$ & 0.0242 \\
\hline $\mathrm{V} \mathrm{b}$ & 14.3 & 11.0 & 3.3 & 10.560 & $\begin{array}{l}\text { R. } 0.497 \\
\text { L. } 0.413\end{array}$ & $\begin{array}{l}0.364 \\
0.413\end{array}$ & $\begin{array}{l}0.203 \\
0.225\end{array}$ & $\begin{array}{l}0.0362 \\
0.0403\end{array}$ & 0.0381 \\
\hline$V_{c}$ & 14.9 & 11.6 & 3.3 & 9.735 & $\begin{array}{l}\text { R. } 0.490 \\
\text { L. } 0.364\end{array}$ & $\begin{array}{l}0.273 \\
0.287\end{array}$ & $\begin{array}{l}0.175 \\
0.168\end{array}$ & $\begin{array}{l}0.0225 \\
0.0177\end{array}$ & 0.0201 \\
\hline $\mathrm{Vd}$ & 12.9 & 11.8 & 1.1 & 10.923 & $\begin{array}{l}\text { R. } 0.602 \\
\text { L. } 0.483\end{array}$ & $\begin{array}{l}0.371 \\
0.371\end{array}$ & $\begin{array}{l}0.217 \\
0.175\end{array}$ & $\begin{array}{l}0.0488 \\
0.0302\end{array}$ & 0.0395 \\
\hline
\end{tabular}


TABLE 1-Concluded

\begin{tabular}{|c|c|c|c|c|c|c|c|c|c|}
\hline \multirow{2}{*}{ NUMBER } & \multirow{2}{*}{$\mid \begin{array}{c}\text { TOTAL } \\
\text { LENGTH }\end{array}$} & \multirow{2}{*}{$\begin{array}{c}\text { BODY } \\
\text { LENGTH }\end{array}$} & \multirow{2}{*}{$\begin{array}{c}\text { TAIL } \\
\text { LENGTH }\end{array}$} & \multirow{2}{*}{$\begin{array}{c}\text { HIND LEG } \\
\text { LENGTH }\end{array}$} & \multicolumn{3}{|c|}{ THYREOID } & \multirow{2}{*}{ VOLUME } & \multirow{2}{*}{$\begin{array}{l}\text { AVERAGS } \\
\text { VOLOMEE }\end{array}$} \\
\hline & & & & & Length & Breadth & $\begin{array}{c}\text { Thick- } \\
\text { ness }\end{array}$ & & \\
\hline \multirow{3}{*}{$\mathrm{Ve}$} & $m m$. & $m m$. & $m m$. & $m m$. & $m m$. & $m m$. & $m m$. & $c m m$. & $c m m$. \\
\hline & 13.8 & 11.9 & 1.9 & 10.890 & R. 0.483 & 0.378 & 0.175 & 0.0310 & \\
\hline & & & & & L. 0.434 & 0.336 & 0.238 & 0.0351 & 0.0480 \\
\hline \multirow[t]{2}{*}{$V \mathbf{f}$} & 14.8 & 11.9 & 2.9 & 10.857 & R. 0.392 & 0.329 & 0.161 & 0.0106 & \\
\hline & & & & & L. 0.409 & 0.315 & 0.182 & 0.0229 & 0.0167 \\
\hline \multirow[t]{2}{*}{$\mathrm{V} \mathbf{g}$} & 12.3 & 11.7 & 0.6 & 11.550 & R. 0.476 & 0.336 & 0.280 & 0.0457 & \\
\hline & & & & & L. 0.409 & 0.357 & 0.287 & 0.0438 & 0.0442 \\
\hline \multirow[t]{2}{*}{$\mathrm{Vh}$} & 13.1 & 11.7 & 1.4 & 9.801 & R. 0.497 & 0.287 & 0.182 & 0.0261 & \\
\hline & & & & & L. 0.532 & 0.336 & 0.231 & 0.0414 & 0.0837 \\
\hline \multirow[t]{2}{*}{$\mathrm{V}$ i } & 12.9 & 11.5 & 1.4 & 9.207 & R. 0.371 & 0.266 & 0.168 & 0.0240 & \\
\hline & & & & & L. 0.427 & 0.294 & 0.196 & 0.0249 & 0.0244 \\
\hline \multirow[t]{2}{*}{$\mathrm{V} \mathrm{j}$} & 13.0 & 11.1 & 2.9 & 10.395 & R. 0.392 & 0.259 & 0.182 & 0.0182 & \\
\hline & & & & & L. 0.364 & 0.287 & 0.182 & 0.0188 & 0.0185 \\
\hline \multirow[t]{2}{*}{ VI a } & & 12.8 & & 10.659 & R. 0.623 & 0.406 & 0.368 & 0.0788 & \\
\hline & & & & & L. 0.567 & 0.413 & 0.287 & 0.0679 & 0.0733 \\
\hline \multirow[t]{2}{*}{$\mathrm{VI} b$} & & 13.5 & & 13.299 & R. 0.616 & 0.385 & 0.287 & 0.0681 & \\
\hline & & & & & L. 0.497 & 0.441 & 0.673 & 0.0594 & 0.0637 \\
\hline \multirow[t]{2}{*}{ VI c } & & 13.8 & & 12.419 & R. 0.651 & 0.392 & 0.294 & 0.0735 & \\
\hline & & & & & L. 0.567 & 0.385 & 0.301 & 0.0650 & 0.0692 \\
\hline \multirow[t]{2}{*}{ VI d } & & 12.9 & & 12.507 & R. 0.413 & 0.308 & 0.294 & 0.0368 & \\
\hline & $\frac{0}{3}$ & & $\frac{8}{0}$ & & L. 0.448 & 0.294 & 0.234 & 0.0287 & 0.0327 \\
\hline \multirow[t]{2}{*}{ VI e } & $\frac{\infty}{5}$ & 12.3 & $\frac{\sqrt[n]{0}}{3}$ & 11.055 & R. 0.658 & 0.448 & 0.294 & 0.0719 & \\
\hline & 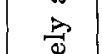 & & 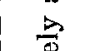 & & L. 0.497 & 0.455 & 0.294 & 0.0667 & 0.0792 \\
\hline \multirow[t]{2}{*}{$\mathrm{VI} \mathrm{f}$} & 莒 & 12.7 & 志 & 10.923 & R. 0.483 & 0.364 & 0.252 & 0.0432 & \\
\hline & 명 & & $\vec{g}$ & & L. 0.511 & 0.399 & 0.301 & 0.0612 & 0.0522 \\
\hline \multirow[t]{2}{*}{ VI $\mathrm{g}$} & $=$ & 12.3 & $\bar{E}$ & 12.474 & R. 0.518 & 0.315 & 0.301 & 0.0484 & \\
\hline & 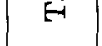 & & & & L. 0.581 & 0.322 & 0.308 & 0.0520 & 0.0502 \\
\hline \multirow[t]{2}{*}{ VI h } & & 11.4 & & 11.088 & R. 0.462 & 0.273 & 0.224 & 0.0273 & \\
\hline & & & & & L. 0.532 & 0.294 & 0.206 & 0.0323 & 0.0298 \\
\hline \multirow[t]{2}{*}{$\mathrm{VI} \mathbf{i}$} & & 11.9 & & 11.979 & R. 0.477 & 0.322 & 0.294 & 0.0445 & \\
\hline & & & & & L. 0.448 & 0.308 & 0.301 & 0.0418 & 0.0431 \\
\hline \multirow[t]{2}{*}{$V I j$} & & 12.2 & & 10.527 & R. 0.504 & 0.357 & 0.294 & 0.0506 & \\
\hline & & & & & L. 0.455 & 0.392 & 0.266 & 0.0474 & 0.0490 \\
\hline
\end{tabular}


The tadpoles selected from a jar containing many hundred were closely matched in six representative stages with ten specimens of each stage. The measurements of lengths of hind leg, body, and total length give a basis for comparing these lots. Of these criteria the most constant is the length of the hind legs. These show a continuous growth, while the total length and body length are modified by the process of metamorphosis, the body showing distinct reduction for a time. The stages chosen may be described as follows:

I. Hind-limb buds very small, the longest showing but faint indications of differentiation into parts. No evidence of fore legs (fig. $1 a$ ).

II. Total length and body length increased. Hind limbs showing differentiation into parts. Toes well differentiated.

III. Hind limbs decidedly larger than in preceding stage. Continued increase in total and body length. Fore limbs formed beneath the skin, but not yet broken through (fig. 2 a).

IV. Continued increase in length of hind limbs. Fore limbs through the skin. Slight decrease in total length and body length.

V. Continued increase in size of limbs. Slight increase in body length, but marked decrease in tail length.

VI. Completion of metamorphosis (fig. $3 a$ ).

In the main there is little need of comment upon the figures in the accompanying tables. In stage I (fig. 4) there is little colloid present in the follicles of the thyreoid glands and many of the follicles are not yet formed, being represented merely by small scattered masses of cells. They lie all in one plane at this time, except in a few cases where they are beginning to arrange themselves in two layers. This process is completed in stage II, where the thyreoid glands show a distinct increase in size and in the number and size of the component follicles (fig. 5). This is continued through later stages. It will be seen that there are many cases where the volume of the thyreoid glands is not proportional to the relative length of the legs or of the body. While this is true in a comparison between the members of group I and group II (with the single exception of II $h$ ), there are no 
members of the former group that have a thyreoid gland volumeapproaching that of any member of group II. The same is true in comparing group II with group III (with the single exception. of II $h$ ). These statements do not hold true, however, in comparing groups $I V, V$, and VI. In these there are a number of cases in which members of a younger stage will show a greater volume of the thyreoid glands than do certain individuals in the higher groups. In fact, the average volume of the thyreoid glands in group $\mathrm{V}$ is less than that of group IV. This point. will be discussed later. Even among the metamorphosed toads, VI $h$, for instance, shows a thyreoid gland volume less than the

TABLE 2

Dimensions of thyreoid glands and body measurements of Bufo lentiginosuslarvae

\begin{tabular}{|c|c|c|c|c|c|c|c|c|c|}
\hline STAGE & $\begin{array}{c}\text { TAIL } \\
\text { LENGTH }\end{array}$ & $\begin{array}{c}\text { TAIL } \\
\text { PROPOR- } \\
\text { TION }\end{array}$ & $\begin{array}{c}\text { BODY } \\
\text { LENGTH }\end{array}$ & $\begin{array}{c}\text { BODY } \\
\text { PROPOR- } \\
\text { TION }\end{array}$ & $\begin{array}{c}\text { LEG } \\
\text { LENGTH }\end{array}$ & $\begin{array}{c}\text { LEG } \\
\text { PROPOR- } \\
\text { TION }\end{array}$ & $\begin{array}{l}\text { THYREOID } \\
\text { VOLUME }\end{array}$ & $\mid \begin{array}{l}\text { THYREOID } \\
\sqrt[3]{\text { VOLUME }}\end{array}$ & $\begin{array}{l}\text { THYREOID } \\
\text { PROPOR- } \\
\text { TION }\end{array}$ \\
\hline & $m m$. & & & & & & & & \\
\hline I & 11.09 & 0.742 & 9.69 & 0.770 & 1.21 & 0.104 & 0.00139 & 0.0518 & 0.0635 \\
\hline II & 13.88 & 0.954 & 11.65 & 0.926 & 3.67 & 0.314 & 0.01270 & 0.5026 & 0.616 \\
\hline III & 14.95 & 1.000 & 12.07 & 0.959 & 7.76 & 0.664 & 0.02612 & 0.6390 & 0.771 \\
\hline IV & 13.63 & 0.912 & 11.13 & 0.885 & 9.17 & 0.785 & 0.03161 & 0.6811 & 0.834 \\
\hline V & 2.91 & 0.195 & 11.58 & 0.920 & 10.49 & 0.898 & 0.03077 & 0.6753 & 0.828 \\
\hline VI & 0.00 & 0.000 & 12.58 & 1.000 & 11.69 & 1.000 & 0.05427 & 0.8158 & 1.000 \\
\hline
\end{tabular}

averages of groups IV and V, while the thyreoid volume of VI $d$ is surpassed by the thyreoid volume of several individuals in each of the two preceding classes. It may be pointed out that this is partially to be explained by the fact that these three stages are really passed through in a relatively short period of time. Figure 6 shows the thyreoid glands of VI $f$.

Table 2 shows the average dimensions of the body and of the thyreoid gland in each of these groups. The actual dimensions. are given, and in the following column is shown, in each case, the proportional size of the feature as compared with the size at. the stage when it shows its maximum development.

Text figure $A$ gives a graphic representation of these features as seen in table 2 . In this case the growth of the hind limbs was taken as a standard for determining the relative stage of devel- 
opment in each group. With the material at hand it was impossible to judge the age of the specimens. This would be quite an unsatisfactory method of seriating material, even in laboratoryreared specimens, because of the large amount of individual variation in the rate of growth of tadpoles. Temperature conditions play a large part in determining the rate of growth. Any attempt to regulate this factor would probably entail abnormal

Relation between thyreoid growth and metamorphosis in Bufo lentiginasus

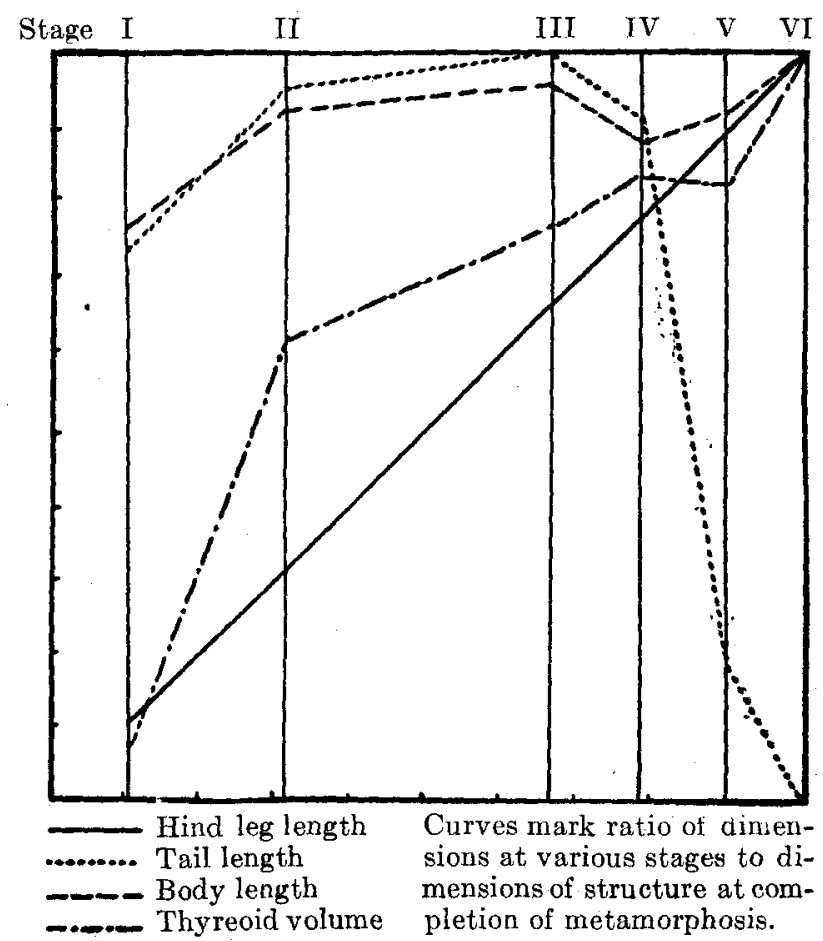

Fig. A Curves to show the relative rate of growth of the total length, body length, and hind-limb length as compared with the growth of the thyreoid gland during metamorphosis in Bufo lentiginosis.

conditions that would modify growth in other ways. It is difficult, at best, to bring about normal development of tadpoles under laboratory conditions. For these reasons it was decided to use specimens caught under natural conditions and seriated as indicated above. 
The growth of the hind limbs was arbitrarily represented by a straight line inclined at an angle of $45^{\circ}$. Upon this were marked off intervals indicating the average length of the hind limbs at the given stage as compared with the average length at the stage of metamorphosis (stage VI). From each of these points a perpendicular was dropped to the base line. These perpendiculars then served to indicate the six stages chosen. Their distance from each other serving to indicate the probable time intervals between the different stages upon the assumption that the growth of the hind limbs takes place at a uniform rate. Points established upon these verticals serve to indicate the average dimensions of various features at each of the six stages studied, the height from the base line showing the proportion that the dimensions of any given feature of that stage bear to its dimensions at the time of metamorphosis-stage VI-maximum development in the case of tail length. Curves were constructed by joining these points, thus giving the proportional rate of growth of each feature. The cube root of the volume of the thyroid gland was employed because it would represent one dimension of a cubical figure whose volume would roughly represent the volume of the thyreoid gland. This appeared to be the best criterion of comparison, because each of the other features was represented by a one-dimension value as body length, tail length, and hind leg length. In reality all of these features have length, breadth, and thickness. Any influence that the thyreoid gland would exert upon their growth would be the influence of one solid body upon another. The length of the thyreoid gland could not be taken as a criterion of comparison, because it increases little during the stages, while the volume of the gland increases greatly, owing to growth in thickness. Thus it seems that the fairest basis of comparison would be to compare the cube root of the volume of the thyreoid with the length dimensions of the body, tail, and hind limb.

It is noted that the cube root of the thyreoid volume shows a marked rise from stage I to stage II, from which the rise continues strongly to stage III, then more strongly to stage IV. There is a slight fall in the curve from stage IV to stage $V$, with 
a sharp rise to stage VI. The body length shows a steady rise to stage III, when it falls off quite distinctly. This is probably due to the shrinkage of the intestine which brings the cloacal opening closer to the root of the tail than it had previously been. It is just at this time that the fore limbs have first appeared. The partly metamorphosed toads are leaving the water at stage $\mathrm{V}$, and an appreciable loss of water from the tissues must take place. However, the body has really begun again to increase in size at stage $V$, and by stage VI it has exceeded the length attained in stage III. The tail reaches its maximum length in stage III, and then rapidly diminishes to the vanishing point.

The cube root of the thyreoid gland increases more rapidly than does the length of the hind legs during the interval between the first and second stages. This is significant in that it corresponds with the results of experimental work which show that the hind limbs develop very slowly in tadpoles from which the thyreoid glands have been extirpated. It is thus seen that growth of the hind legs is to a very large extent dependent upon the growth of the thyreoid gland.

A study of sections of the thyreoid glands shows that colloid begins to form at about the time when the hind limbs commence to develop. Compare figures $1 b, 2 b$, and $3 b$ and table 3 . It increases in amount as growth continues. Measurements of the diameter of the larger colloid masses at different stages of development show a steady increase in size, very rapid in the early stages, as seen in table 3 . In each specimen micrometer measurements were made of ten of the larger colloid masses. An average was then calculated in each case (table 3 ).

The colloid masses increase in size until stage III, while in $\mathrm{V}$ there is a diminution in size. This is observed even in some of the metamorphosed toads, while in others the colloid masses have reached a size beyond that found in stage III. This table is clearly based upon too few observations to prove in itself of much value. It is of significance, however, in that it corresponds in a general way with the results of table 1 . We see that the increase in size of the thyreoid gland corresponds with an increase in size of the colloid masses. It appears that this 
growth of the glands is, in fact, brought about by the accumulation of colloid substance. In the first stages of colloid formation there were only from five to ten colloid masses. These soon became very numerous, as shown in figures $1 b, 2 b$, and $3 b$. No satisfactory conclusions were drawn from a study of the epithelium of the follicles, although it is quite possible that important points might be gained by an application of special methods of technique upon the problem of the manner in which they elaborate the thyreoid secretion.

TABLE 3

Table of measurements of colloid masses in the thyreoid gland of Bufo lentiginosus

\begin{tabular}{|c|c|c|c|}
\hline TOTAL LENGTH & BODY LENGTH & HIND-LEG LENGTH & $\begin{array}{l}\text { AVERAGE COLLOID } \\
\text { DIAMETER }\end{array}$ \\
\hline$m m$. & $m m$. & $m m$. & $m m$. \\
\hline 8.4 & 3.4 & & \\
\hline 8.4 & 3.7 & & No colloid present \\
\hline 8.9 & 3.8 & & \\
\hline 9.1. & 4.3 & & \\
\hline 11.4 & 5.5 & 0.245 & 0.0122 \\
\hline 14.6 & 6.9 & 0.357 & 0.0138 \\
\hline 15.1 & 8.4 & & 0.0147 \\
\hline 20.8 & 9.5 & & 0.0284 \\
\hline 20.8 & 10.6 & 2.805 & 0.0258 \\
\hline 23.6 & 11.8 & 4.884 & 0.0559 \\
\hline 24.8 & 11.4 & 6.270 & 0.0603 \\
\hline 13.1 & 10.1 & 7.623 & 0.0499 \\
\hline 12.0 & 12.0 & 10.164 & 0.0478 \\
\hline 12.3 & 12.3 & 11.055 & 0.0672 \\
\hline
\end{tabular}

\section{SUMMARY AND CONCLUSIONS}

The accumulation of colloid material in the thyreoid glands of toad tadpoles begins just as the hind limb buds appear. The colloid masses continue to increase in size and number until the fore limbs break through the skin. This accumulation of colloid material is accompanied by a marked increase in the size of the thyreoid glands, which appears in the main to be a direct result of it.

A series of observations upon the effect of thyreoid extirpation in Rana and Bufo upon limb development have shown that the 
limb buds appear simultaneously in both the control and operated tadpoles. Soon after their appearance the limb buds of the thyreoidless tadpoles lag far behind those of the normal controls. They finally grow to an appreciable degree in spite of the absence of the thyreoid gland, but never so fast nor to any degree approaching the length relative to body length attained in the normal controls. These observations will be extended during the coming season and published later. It is clear, however, that the effects of thyreoid removal first become evident in Bufo at the period when colloid normally begins to accumulate. A comparative study along this line would give some valuable hints upon the real significance of colloid secretion and accumulation.

We have next to consider the apparently paradoxical fact that there is a cessation in growth and an actual diminution in the size of the thyreoid glands and of the colloid masses at the very time when the process of metamorphosis is most active (stage V). This might in part be explained as the result of a partial drying process due to the emergence of the tadpoles from the water, were it not for the fact that they do not emerge upon the land until stage $V$. The reduction in size is thus under way before this factor could prove effective. It is much more probable that this diminution may be due to the absorption of an unusually large amount of stored colloid at this time when it would prove most effective. It is quite conceivable that substances might be elaborated in the blood that would enable it to more readily dissolve the colloid and that its solvent power might decrease again after metamorphosis has been completed. Of course this is pure conjecture, but it is put forth in the hope that it may prove suggestive.

The development of the tail presents an interesting problem. It steadily increases in size until stage III, shows a slight diminution to stage IV, and then quickly disappears. It might be assumed that a certain amount of thyreoid secretion must be elaborated before the absorption of the tail can be accomplished, or, if our assumption of a more solvent condition of the blood should prove true, it might serve to explain this point. What- 
ever the means by which it is accomplished, we should have to choose between two alternatives: either the thyreoid secretion does not cause the shrinkage of the tail, or it must reach a considerable volume before it is able to accomplish that result. It is certain that limb development and the process of disappearance of the tail follow the accumulation of colloid in the thyreoid glands of Bufo.

\section{BIBLIOGRAPHY}

AdLer, Leo 1914 Metamorphosestudien an Batrachierlarven. I. Exstirpation endokriner Drusen. B. Exstirpation der Thymus. Archiv $f$. Entw.-Mech. d. Organismen, Bd. 40.

Allen, B. M. 1916 The results of the extirpation of the anterior lobe of the hypophysis and of the thyroid of Rana pipiens larvae. Science, Nov. 24th.

1918 The results of thyroid removal in the larvae of Rana pipiens. Jour. Exp. Zool., vol. 24.

Goetre, A. 1875 Entwickelungsgeschichte der Unke. Leipzig, 1875.

Godernatsch, J. F. 1912 Fütterungsversuche an Amphibienlarven. Centralbl, f. Physiologie, 1912.

1912 Feeding experiments on tadpoles, etc. Arch. f. Entw.-Mech., Bd. 35.

MAORER, F. 1888 Schilddrüse, Thymus und Kiemenreste der Amphibien. Morph. Jahrb., Bd. 13.

Medron, D. DE 1886 Recherches sur le développement du thymus et de la glands thyreoide. Receuil Zool. Suisse, 1 Ser. V. 3.

MüLler, W. 1871 Uber die Entwicklung der Schilddrüse. Jenaische Zeitschr., Bd. 6.

Hoskins, E. R. ANd Margarep Morris 1917 Thyroidectomy in Amphibia. Anat. Rec., vol. 11.

Rogers, JAMES B. 1918 The effects of the extirpation of the thyroid upon the thymus and the pituitary glands of Rana pipiens. Jour. Exp. Zool. vol. 24.

Swingle, W. W. 1918 The acceleration of metamorphosis in frog larvae by thyroid feeding and the effects upon the alimentary tract and sex glands. Jour. Exp. Zool., vol. 24.

Terry, George S. 1918 Effects of the extirpation of the thyroid gland upon ossification in Rana pipiens. Jour. Exp. Zool., vol. 24. 
PLATE 
PLATE 1

\section{EXPIANATION OF FIGURES}

1a, 2a, and 3a Drawings to show typical stages in the development of Bufo lentiginosus. Figure 1a represents stage I. Figure $2 \mathrm{a}$ represents stage III. Figure 3a represents stage VI. All drawn to scale and magnified $\times 3$.

$1 \mathrm{~b}, 2 \mathrm{~b}$, and $3 \mathrm{~b}$ Drawings to show transverse sections of the thyreoid glands of $1 \mathrm{a}, 2 \mathrm{a}$, and $3 \mathrm{a}$, respectively. Figure $1 \mathrm{~b}$, transverse section of the thyreoid gland of $1 \mathrm{a}$. Figure $2 \mathrm{~b}$, transverse section of the thyreoid gland of 2a. Figure $3 \mathrm{~b}$, transverse section of the thyreoid gland of 3a. All drawn to scale and magnified $\times 75$.

4,5 , and 6 Whole mounts of the thyreoid glands of the stages represented above. Figure 4, whole mount of thyreoids of $I \mathrm{~b}$. Figure 5 , whole mount of thyreoids of II $h$. These thyreoids have a volume almost identical with the average in group III. Figure 6, whole mount of thyreoids of VI f. All drawn to scale and magnified. 


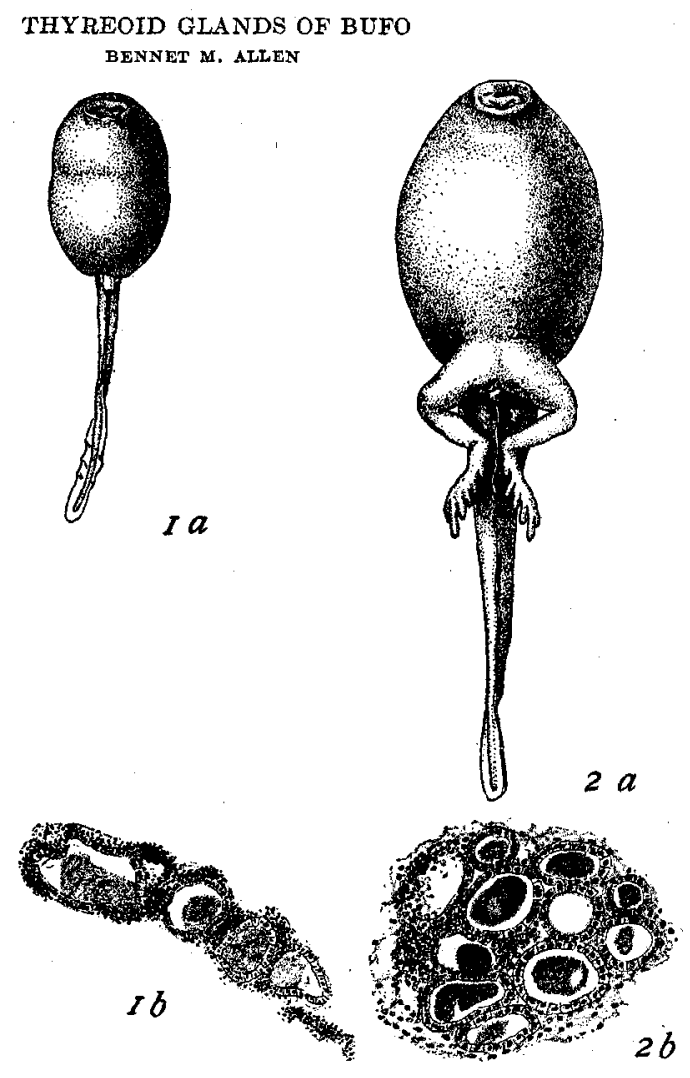

PLATE 1
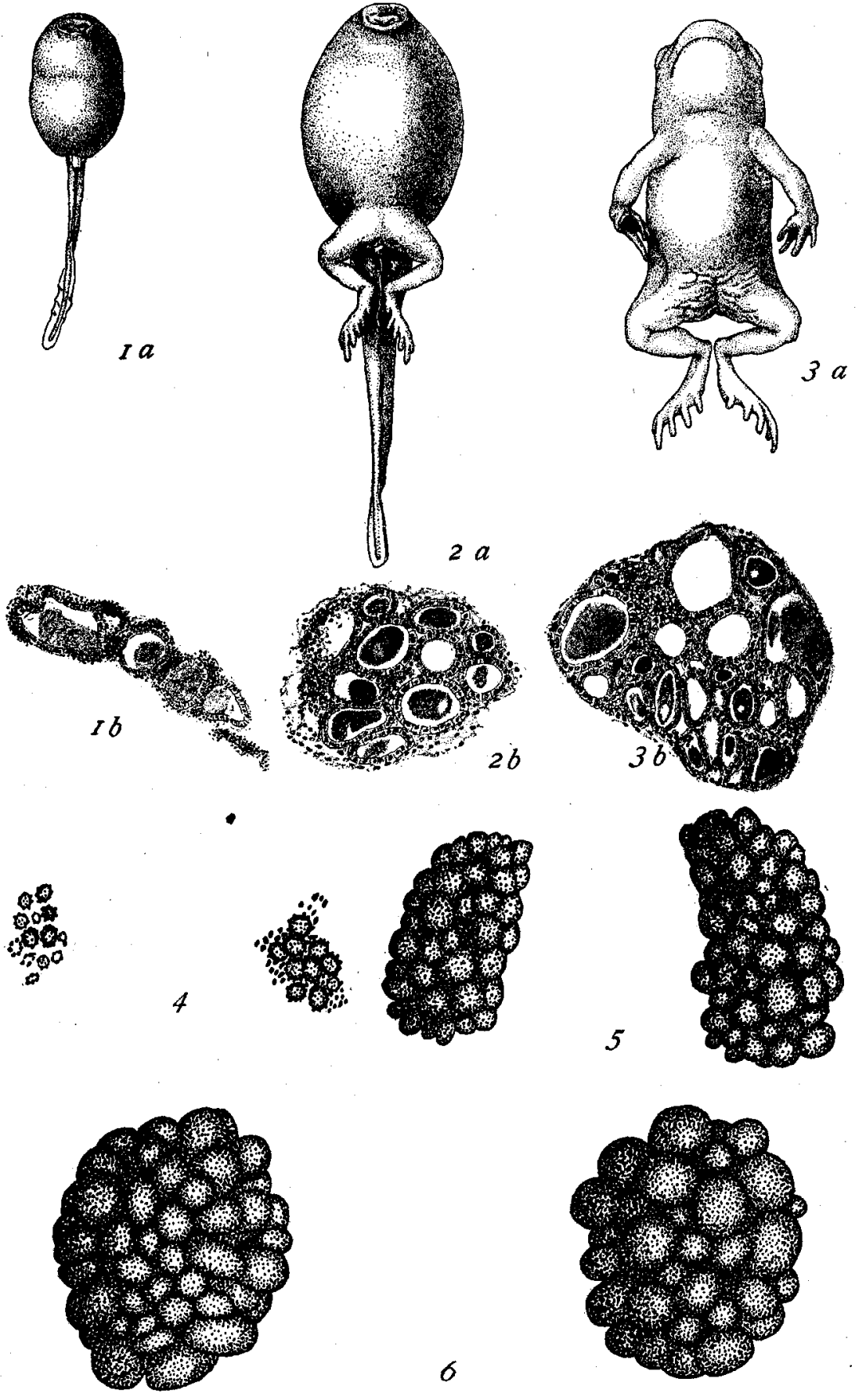

6

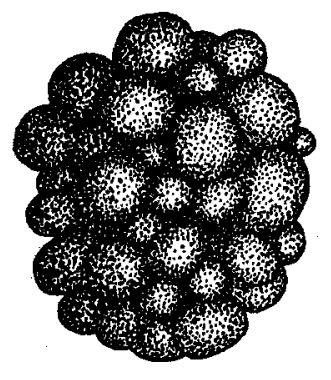

